\title{
Assessment of ovarian tumor marker CA-125 during radiotherapy course
}

\author{
Mohammed A. Ali Omer ${ }^{\text {ab*}}$, Mohamed Elfadil Mohamed ${ }^{a}$, Mohamed Yousef ${ }^{\mathrm{b}}$, Mohamed Farahna ${ }^{\mathrm{b}}$ \\ ${ }^{a}$ College of Medical Radiologic Science, Sudan University of Science and Technology - Khartoum, Sudan. \\ ${ }^{b}$ Radiologic Technology Department, College of Applied Medical Science, Qassim University-Buraidah, KSA.
}

ARTICLE INFO ABSTRACT

\section{Article History}

Received $\quad 12 / 05 / 2013$

Accepted $\quad 16 / 05 / 2013$

\section{* Correspondence to:}

Mohammed A. Ali Omer

College of Medical Radiologic Science,

Sudan University of Science and

Technology, Khartoum, Sudan.

e-mail: alkajam@gmail.com

\begin{abstract}
The antigen secretion by ovarian cancer (CA-125) has been assessed using Radio Immune Assay (RIA) technique among a hundred ovarian cancer patients who received radiotherapy course as radical treatment. The study revealed that, the ovarian cancer commonly associated with the age groups of 30-40 years old taking a percent of 68 relative to other age groups and the antigen CA-125 does not has relation with aging. While the radiotherapy shows it is efficiency in management of ovarian cancer by reducing the antigen CA-125 level from $134 \pm 13.3 \mathrm{U} / \mathrm{ml}$ to $5.5 \pm 2.1 \mathrm{U} / \mathrm{ml}$ relative to normal level of CA-125 (35 U/ml). Also there is significant relationship between the cancer stage and the level of antigen CA-125 $(\mathrm{R} 2=0.98)$.
\end{abstract}

J. Exp. Clin. Med., 2013; 30:137-139

Keywords:

Ovarian cancer

Radiation therapy and tumor maker

Radioimmuneassay

\section{Introduction}

The ovaries are female's reproductive organs and endocrine glands as well, to produce ovum (Goone, 2007; National Comprehensive Cancer Network, 2009) and secrete both estrogen and progesterone.

Like other organs, ovaries can be involved by cancer which is defined as: (Medical term: Malignant neoplasm) a class of diseases in which a group of cells display uncontrolled growth, invasion that intrudes upon and destroys adjacent tissues, and sometimes metastasis, or spreading to other locations in the body via lymph or blood. These three malignant properties of cancers differentiate them from benign tumors, which do not invade or metastasis (Yin and Lloyd, 2001).

The ovarian cancer is the fifth most common cancer among women, and it causes more deaths than any other type of female reproductive cancer (Goone, 2007). Such cancerous disease could be diagnosed by different modalities, such as blood tests, X-rays, CT scans and endoscopy (Goone, 2007), in addition to radioimmunoassay (RIA) (Bast et al, 1983) which is dealing with assessment of hormonal assay level that related to specific malignant growth in human body known as tumor marker or tumor antigens.
In this realm, several antigens have been detected in association with ovarian carcinomas (Donaldson et al., 1980; Bhattacharya et al., 1982) and of this CA-125 have shown the most clinical promise to date (Bast et al., 1983; Canney et al., 1984). The antigen CA-125, has high molecular weight glycoprotein expressed in coelomic epithelium during embryonic development, is defined by a murine monoclonal antibody raised against a serous ovarian carcinoma cell line; in addition to Human Epididimis Protein 4 (HE4) which is a newly tumor marker for ovarian cancer that belongs to a group having four disulfide core proteins. It is first identified in the epithelium of distal epididimis and thought to be a protease inhibitor that took part in sperm maturation (Manolov et al, 2012).

The most widely used tumor marker in ovarian cancer, often considered as the gold standard is the antigen CA-125 (Hogdall, 2008). It was first identified by Bast, Knapp, and colleagues in 1981. CA-125 is used at the time of diagnosis of the disease, to evaluate the possibility of complete resection during surgery, to estimate sensibility for adjuvant or neo-adjuvant chemotherapy and for diagnosis of recurrences (Coussy et al, 2011). 
Antigen CA-125 is the standard tumor marker used to follow women during or after treatment for epithelial ovarian cancer (the most common type of ovarian cancer). Normal blood levels are usually less than $35 \mathrm{U} / \mathrm{ml}$. More than $90 \%$ of women have high levels of antigen CA-125 when the cancer is advanced. If the antigen CA-125 level is increased at the time of diagnosis, but in only $50 \%$ of patients with stage I disease, changes in the antigen CA-125 level can be used during treatment to get an idea of how well it's working.

\section{Methods}

The blood samples as three ml were obtained from 100 patients who had been referred to the Radiation and Isotopes Center of Khartoum for treatment of primary ovarian carcinoma. The blood samples were centrifuged and left for three hours to insure that the plasma is totally separated from the serum. The serum of ovarian tumor marker (CA-125) had been estimated as described By Tietz, (1995) and ACOG, (2002), before starting radiotherapy course, after finishing the radiotherapy course, and for different stages of ovarian carcinoma (stage I, II, III and IV) for all patients sample which consists of 100 patients.

The tumor staging (stage I, II, III and IV) were carried out according to FIGO classification as described in National Comprehensive Cancer Network (NCCN, 2009).

\section{Results}

The following results are dealing with a sample consist of 100 ovarian cancer patients who received external radical radiotherapy. The collected data analyzed and the obtained results are as following (Fig. 1-5).

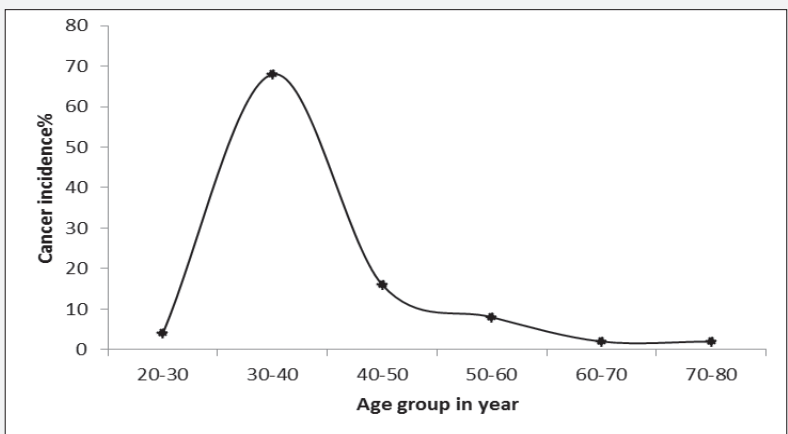

Fig. 1.The incidence percent of ovarian carcinoma in age's groups

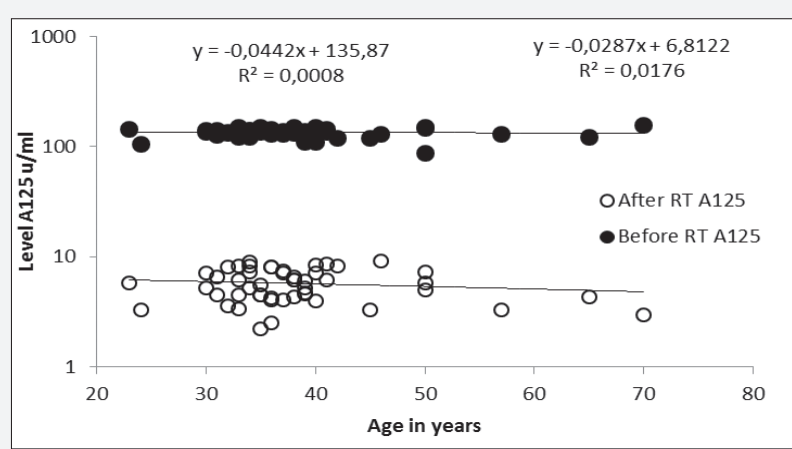

Fig. 2. The correlation between levels of CA-125 versus age prelpost radiotherapy.

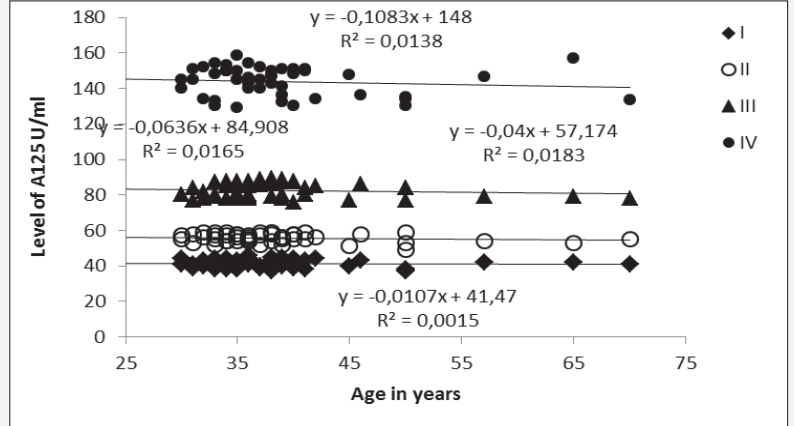

Fig. 3. The correlation between the level of CA-125 and age for different cancer stages.

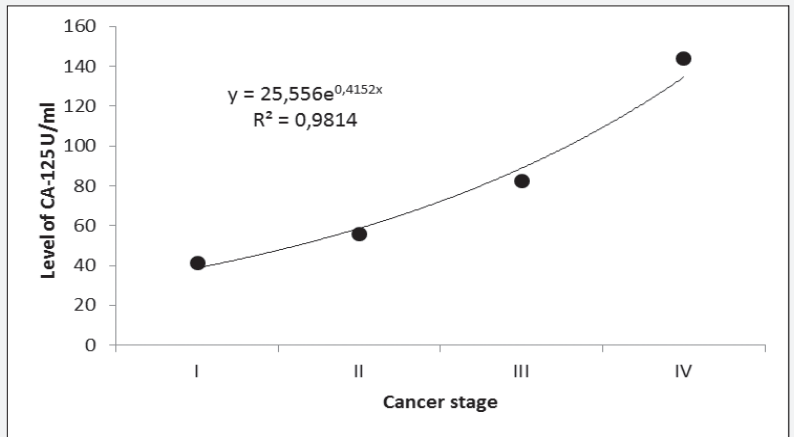

Fig. 4. The correlation between the level of CA-125 and the stage of ovarian cancer

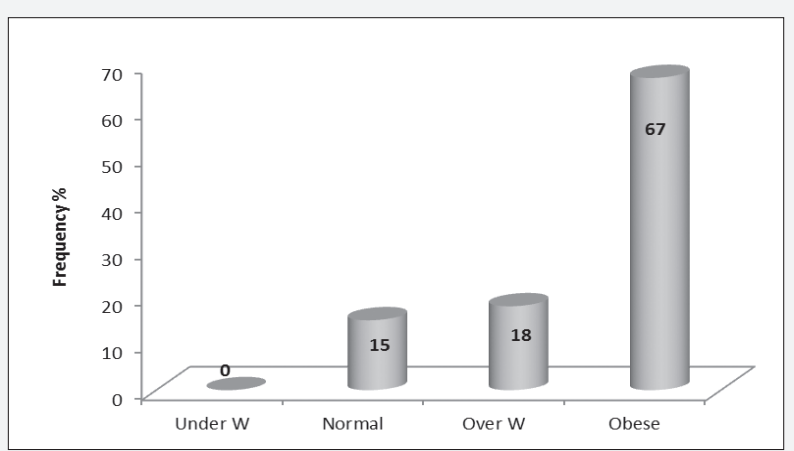

Fig. 5. The distribution of cancer patients based on body mass index

\section{Discussion}

Figure 1 shows the incidence percent of ovarian carcinoma in age's groups. It revealed the common involved age by ovarian carcinoma was the age group of 30-40 years old taking a percent of 68 relative to other age groups; however the age group of 40-50 years old was also showed considerable incidence scoring $16 \%$. The high incidence of ovarian carcinoma in these ages group could be ascribed to obesity of the patients as well as the common types of histology as has been reported by Kramer and Greene, (2004) and American Cancer Society, (2012).

Figure 2 shows the correlation between levels of CA-125 versus age prelpost radiotherapy. It deduced that the correlation of antigen CA-125 versus age was so insignificant prel post radiotherapy course $(\mathrm{R} 2=0.00$ and $\mathrm{R} 2=0.02)$ respectively. And the correlation could be fitted in the form of linear equa- 
tion: Where Y refers to level of CA-125 and $\mathrm{x}$ refers to age in years. While after radiotherapy course the result showed a significant effect of radiotherapy in ovarian carcinoma based on the level of antigen CA-125 before and after irradiation. As the average of CA-125 before radiotherapy was $134 \pm$ $13.3 \mathrm{U} / \mathrm{ml}$ relative to $5.5 \pm 2.1 \mathrm{U} / \mathrm{ml}$ after radiotherapy i.e. there was $128.5 \mathrm{U} / \mathrm{ml}$ of antigen CA-125 difference which is equivalent to $367.1 \%$ relative to normal level of CA-125 ( $35 \mathrm{U} / \mathrm{ml}$ ). These results strengthen the study of Sproston et al., (1995) in which they showed that the CA-125 serum is important for assessing prognosis of tumor especially ovarian carcinoma.

Figure 3 shows the correlation between the level of CA125 and age for different cancer stages. It showed that there was no significant effect of aging in the level of CA-125 as the mean significant coefficient $(\mathrm{R} 2=0.01)$. However there was considerable increasing effect of staging in the level of CA-125, for stage I, II, III and IV the average level of CA-125 was 41.1, 55.6, 82.5 and 143.8 respectively. Such results have been reported by Rustin et al., 1996, 1999. Figure 4 shows the correlation between the level of CA-125 and the stage of ovarian cancer. It shows that there is significant increasing effect of cancer stage on the level of the CA-125 giving a significant correlation coefficient at $(\mathrm{R} 2=0.98)$, and the relation shown in an exponential equation of the form of: where $y$ refers to the level of CA-125 and $\mathrm{x}$ refers to the stage of ovarian cancer. The increasing of CA-125 in U/ml by advancing in the cancer stage is ascribed to exponential proliferation of cancer tissues.

Figure 5 shows the distribution of cancer patients based on body mass index (BMI). The BMI formula or (Quetelet Index) which developed by Belgium statistician Adolphe Quetelet (1796-1874) (Keys et al, 1972), as an international method to measure the obesity as (normal range 18.5-24.9, overweight 25-29.9 and obese is $\geq 30$ ), has been applied and showed that the average BMI of the patients was 33.2 indicating that most of the patients were obese ( $67 \%$ of the sample) hence they were susceptible to ovarian cancer development as has been reported by Gregory et al, (2006) and Ovarian Cancer National Alliance, (2004) that obesity is a cofactor for ovarian carcinoma.

\section{REFERENCES}

ACOG: American College of Obstetricians and Gynecologists, 2002. The role of the generalist obstetrician-gynecologist in the early detection of ovarian cancer. Obstet. Gynecol. 100, 1413-1416.

American Cancer Society. Cancer Facts and Figures. (2012). Atlanta, GA.

Bast, R.C. Jr., Feeney, M., Lazarus, M., Nadler, L.M., Colvin, R.B., Knapp, R.C., 1981. Reactivity of a monoclonal antibody with human ovarian carcinoma. J. Clin. Invest. 68, 1331-1337.

Bast, R.C. Jr., Klug, T.L., ST. John, E., 1983. A radioimmunoassay using a monoclonal antibody to monitor the course of epithelial ovarian cancer. N. Engl. J. Med., 308, 883-887.

Bhattacharya, M., Chatterjee, S.K., Barlow, J.J., Fuji, H. 1982. Monoclonal antibodies recognizing Tumour-associated antigens of human ovarian mucinous cystadenocarcinomas. Cancer Res. 42, 1650-1654.

Canney, P.A., Moore M., Wilkinson, P.M., James, R.D., 1984. Ovarian cancer antigen CA125: A prospective clinical assessment of its role as a tumor marker. Brth. J. Cancer. 50, 765-769.

Coussy, F., Chéreau, E., Daraï, E., Dhombres, F., Lotz, J.P., Rouzier, R., Selle, F., 2011. Interest of CA 125 level in management of ovarian cancer. Gynecol. Obstet. Fertil. 39, 296-301.

Donaldson, E.S., Van Nagell, J.R., Pursell, S., 1980. Multiple biochemical markers on patients with gynaecologic malignancies. Cancer. 45, 948-953.

Goone Wardene, T.I., 2007. Management of asymptomatic patients on follow-up for ovarian cancer with rising CA-125 concentrations. Lancet Oncol. 8, 813-821.

Gregory, P. Beehler., Manveen S., Julie, A. B., Barbara E. T., Susan, E. M., Kerry J. R., and Kirsten B. M., 2006. Risk of ovarian cancer associated with BMI varies by menopausal status.J. Nutr. 136, 2881-2886.

Hogdall, E., 2008. Cancer antigen CA-125 and prognosis. Curr. Opin. Obstet. Gynecol. 20, 4-8.

Keys, A., Fidanza, F., Karvonen, M.J., Kimura, N., Taylor, H.L., 1972. Indices of relative weight and adiposity. J. Chronic. Dis. $25,329-343$.

Kramer, J.L., Greene, M.H., 2004. Epidemiology of Ovarian, Fallopian Tube, and Primary Peritoneal Cancers. in: Gynecologic Cancer: Controversies in Management. Gershenson, D., Gore, M., McGuire, W., Quinn, M., Thomas, G., editors. Elsevier Science. pp. $327-340$.

Manolov, V., Marinov, B., Vasilev, V., 2012. HE4 and CA125 in ovarian cancer. Akush. Ginekol. 51, 21-28.

NCCN: National Comprehensive Cancer Network, 2009. NCCN Practice Guidelines in Oncology: Ovarian cancer, Vol. 2.

Rustin, G.J.S., Nelstrop, A.E., Bentzen, S.M., Piccart, M.J., Bertelsen, K., 1999. Use of tumour markers in monitoring the course of ovarian cancer. Ann. Oncology. 10, 21-27.

Rustin, G.J.S., Nelstrop, A.E., Tuxen, M.K, Lambert, H.E., 1996. Defining progression of ovarian carcinoma during follow-up according to CA-125: A North Thames Ovary Group Study. Ann. Oncology. 7, 361-364.

Sproston, A.R.M., Roberts, S.A., Davidson, S.E., Hunter, R.D., West, C.M.L., 1995. Serum tumor markers in carcinoma of the uterine cervix and outcome following radiotherapy. Brith. J. Cancer. 72,1536-1540.

Tietz, N.W. (Ed)., 1995. Clinical guide to laboratory tests, $3^{\text {rd }}$. ed. W. B. Saunders, Philadelphia, PA .pp.

Yin, B.W., Lloyd, K.O., 2001. Molecular cloning of the CA125 ovarian cancer antigen: Identification as a new mucin, MUC16. J. Biol. Chem. $276,27371-27375$ 